\title{
Improvement of Outcome by Implementation of Enhanced Recovery Pathway in Gynecologic/ Oncology Surgery
}

Abdel Magid Mahmoud Sarhan', Hussein Mohammed Abdel Dayem', Heba Mohamed Fathy Abdelrahman ${ }^{2}$, Radwa Atef Ameen Elnaem ${ }^{1}$

Departments of ${ }^{1}$ Obstetrics and Gynecology and ${ }^{2}$ Anesthesia Intensive Care, Faculty of Medicine, Zagazig University, Egypt

*Corresponding Author: Radwa Atef Ameen Elnaem, Mobil: (+20)01090722771, E-mail: drradwaatef9025@ yahoo.com

\begin{abstract}
Background: Enhanced recovery programs (ERPs) are evidence-based protocols designed to improve functional rehabilitation after surgery. They lead to improvement in patient outcomes while reducing length of hospital stay. Objective: This study aimed to compare surgical outcomes between women undergoing major gynecologic surgeries before and after implementation of enhanced recovery after surgery (ERAS) protocol.

Patients and Methods: This erective observational study was carried out on patients attending to Zagazig University Hospitals for Elective Gynecological Oncologic Surgeries in the period between August 2018 and August 2019.

Patients and methods: This study included 54 patients who were presenting for elective gynecological oncologic surgeries. They were classified into 2 groups. Group 1 include 27 patients who were exposed to the ERAS protocol regimen for the pre, intra and postoperative care. Group 2 include 27 patients who were exposed to the conventional care known in the literature. Clinical outcomes and compliance were obtained using the ERAS Interactive Audit System. Results: This study showed that the mean length of hospital stay was $38.29 \pm 4.95$ hours in group A and $68.44 \pm 6.5$ hours in group B with significant difference between the two groups. Postoperative complications rate was $7.4 \%$ in group A vs $11.1 \%$ in-group B with no significant difference between the two groups.
\end{abstract}

Conclusions: Systematic implementation of ERAS gynecologic oncology guidelines across a healthcare system improves patient outcomes and saves resources.

Keywords: Enhanced recovery programs (ERPs), Improvement of outcome, Gynecologic oncology.

\section{INTRODUCTION}

The enhanced recovery after surgery concept emerged as a multimodal approach directed at optimizing the patient experience, standardizing perioperative care, and improving surgical outcomes ${ }^{(\mathbf{1})}$.

Enhanced recovery programs (ERPs) are evidencebased protocols designed to improve functional rehabilitation after surgery. They lead to improvement in patient outcomes while reducing length of hospital stay. Over the previous decade, important progress has been achieved in both benign and oncologic gynecologic surgery, including further refinement of minimally invasive surgery, introduction of the sentinel lymph node concept, individualized lymphadenectomy for endometrial cancer, and adopting of optimal cytoreduction to no visible residual disease for patients with ovarian cancer. These practice changes reduced surgical morbidity, shortened recovery, and improved oncologic outcomes ${ }^{(2-4)}$.

Key components of enhanced recovery protocols include comprehensive patient education including patient goals and 1) expectations around surgery, 2) markedly diminished duration of the fasting period preoperatively and active use of oral carbohydrate and electrolyte fluids up until presentation for surgery, 3) multimodal pain control regimen including nonopioid analgesic agents and regional anesthesia to reduce opioid use, and 4) quick resumption of a normal diet and activity. Trials of these protocols have been

performed in a myriad of surgical settings and have shown, almost uniformly, improved results such as decreased length of hoepital stay and improved surgical outcomes ${ }^{(5,6)}$.Individual components of perioperative care have increasingly been evaluated from an evidence-based perspective, resulting in the creation of what so-called "enhanced recovery" pathways (ERP) ${ }^{(7)}$. This study aimed to compare surgical outcomes between women undergoing major gynecologic surgeries before and after implementation of ERAS protocols.

\section{METHODS}

After obtaining approval of the ethics committee, This ereceptive observationally study was carried out on patients attending Zagazig University Hospitals for Elective Gynecological Oncologic Surgeries in the period between August 2018 and August 2019. This study included 54 patients who were presenting for elective gynecological oncologic surgeries. They were classified into 2 groups. Group 1 include 27 patients were exposed to the ERAS protocol regimen for the pre, intra and postoperative care. Group 2 include 27 patients were exposed to the conventional care known in the literature. Patients' age ranged from 23-80 years old in both groups.

\section{Ethical Approval:}

The work was approved from Ethical Committee, Faculty of Medicine, Zagazig University. The study was carried out in accordance with the World Medical Association's Code of Ethics (Helsinki Declaration). 
Inclusion criteria: Patients who are referred for hysterectomy and/or pelvic or para-aortic lymphadenectomy for gynecological cancer (cervical, endometrial, ovarian, other including borderline ovarian tumor, endometrial hyperplasia and cervical intraepithelial neoplasia).

Exclusion criteria: Patients with bowel resection. Patients with intestinal injuries. Post-operative admission to intensive care unit (ICU) for more than one night.

All patients were subjected to the following: Thorough clinical evaluation with emphasis on full medical and surgical history. General clinical examination and laboratory investigations according to the type of the cancer and suggestions of committee of Multidisciplinary team of Zagazig gynecological and obstetrics unit. Complete blood count (CBC), liver function tests, kidney function test, coagulation profile, random blood sugar, viral markers $(\mathrm{HBV}, \mathrm{HCV})$, tumor markers (CA 125, CEA, CA 199 and Alpha Fetoprotein) (in cases of ovarian cancer). Radiological studies including pelvi-abdominal ultrasound, MRI with dye examination on the pelvis, chest $\mathrm{x}$-ray and CT with contrast. Electrocardiogram, upper and lower endoscopy and cystoscopy if indicated.

\section{Techniques:}

\section{Day before surgery:}

The study group decreased the fasting period, given fluids rich in carbohydrates. The control group completed fasting up to 12 hours for solids and fluids from the time of the operation. No carbohydrate loading preoperatively.

\section{Day of surgery:}

The study group preemptive analgesia was multimodal analgesic that included celecoxib $200 \mathrm{mg}$ PO, gabapentin $600 \mathrm{mg}$ PO, and acetaminophen $975 \mathrm{mg}$ PO. Bowel preparation: no systemic use of mechanical bowel preparation while rectal enemas still used. Prewarming the patient with blankets prior entry to the operating room to increase core temperature prior to surgery.

The control group did not receive preemptive analgesia preoperatively. Mechanical bowel preparation is used.

\section{Intra operative}

All patients of both groups used general anesthesia. Using of short-acting volatile anesthetics or continuous infusion of propofol was recommended to allow rapid surfacing of anesthesia. Avoidamce of administration of NG tube and its removal at the end of operation if used. Pre warming of fluids before infusion during operation to maintain normothermia. Antibiotic prophylaxis: patients received cefotax ( $1 \mathrm{~g}$ or $2 \mathrm{~g} \mathrm{IV})$ before skin incision. Maintainence of intraoperative euvolemia by decreasing crystalloid administration and increasing colloid if needed. Administration of a prophylactic for postoperative nausea and vomiting: dexamethasone $4 \mathrm{mg}$ IV once plus droperidol $0.625 \mathrm{mg}$
IV once half hour before incision, and granisetron 0.1 $\mathrm{mg}$ IV once half hour before incision closure. Use of opiates if needed.

The study group: After incision closure; injection of bupivacaine at incision site. Injection of ketorolac 15 $\mathrm{mg}$ IV at the end of the operation for patients who tolerate it. Trying to limit prophylactic peritoneal drains. Use minimally invasive surgical techniques.

\section{Postoperative:}

All patients of both groups were askede for early strolling. Administration of thromboembolism prophylaxis; enoxaparin $40 \mathrm{mg}$ SC with sequential compression devices. Patients received clear fluids in the recovery room as tolerated and received a soft diet on PO day 1. IV fluids decreased to $40 \mathrm{ml} / \mathrm{h}$ on POD 0 and stopped at 8:00 AM on POD 1. Early removal of catheter as early as possible immediately after surgery or after 6 hours postoperatively. The patient got out of bed for 2 hours at least on the day of the surgery and then 6 hours per day until discharge. Antibiotics were given after 12 hours of exit from the surgery. Pain score was assessed using Universal Pain Tool after complete recovery. The patient discharge criteria included tolerating diet, ambulatory, and pain well controlled on oral analgesia.

\section{Statistical Analysis}

Data collected throughout history, basic clinical examination, laboratory investigations and outcome measures were coded, entered and analyzed using Microsoft Excel software. Data were then imported into Statistical Package for the Social Sciences (SPSS version 20.0) software for analysis. According to the type of data, qualitative data were represented as number and percentage.

Quantitative data were represent by mean \pm SD. The following tests were used to test differences for significance: Chi square test $\left(\mathrm{X}^{2}\right)$ for difference and association of qualitative variable. Differences between quantitative independent groups by $\mathrm{t}$ test. $\mathrm{P}$ value was set at $\leq 0.05$ for significant results $\&<0.001$ for high significant result.

\section{RESULTS}

This study showed that age was distributed as $51.25 \pm 16.1$ and $56.55 \pm 11.94$ years and BMI was $26.09 \pm 2.19$ and $25.54 \pm 2.36$ for study \& control group respectively with no significant difference between both groups. Regarding parity, majority of both group were $>2$ with no significant difference between groups (Table 1). Table (2) showed that there was no significant association or difference between both groups regarding pathology finding distribution.

Moreover, there was no significant association or difference between both groups regarding complications and outcome distribution (Table 3).

There was no significant difference between groups regarding HB or PLT as they were distributed as 
$11.82 \pm 0.56 \& 11.74 \pm 0.71$ and $236.74 \pm 30.3 \& 248.96$ \pm 38.9 respectively (Table 4 ).

Table (5) showed that ERP group had significantly shorter durations regarding catheterization period, operative time, movement time, hearing sounds, oral intake and time until discharge.
ERP significantly needed less opiate (Table 6).

Pain was significantly lower at ERP group but satisfaction was significantly higher at ERP group $(\mathrm{P}<$ $0.05)$ as shown in table (7).

Table (1): Demographic and obstetric characters distribution between groups

\begin{tabular}{|c|c|c|c|c|c|}
\hline & & & ERP Group (N=27) & Control Group $(\mathrm{N}=27)$ & $\mathbf{P}$ \\
\hline \multicolumn{3}{|l|}{ Age } & $51.25 \pm 16.1$ & $56.55 \pm 11.94$ & 0.176 \\
\hline \multicolumn{3}{|l|}{ BMI } & $26.09 \pm 2.19$ & $25.54 \pm 2.36$ & 0.376 \\
\hline \multirow{8}{*}{ Gravidity } & \multirow{2}{*}{ Virgin } & $\mathbf{N}$ & 6 & 0 & \multirow{8}{*}{0.074} \\
\hline & & $\%$ & $22.2 \%$ & $0.0 \%$ & \\
\hline & \multirow{2}{*}{ P 0} & $\mathbf{N}$ & 5 & 5 & \\
\hline & & $\%$ & $18.5 \%$ & $18.5 \%$ & \\
\hline & \multirow{2}{*}{$1-2$} & $\mathbf{N}$ & 3 & 4 & \\
\hline & & $\%$ & $11.1 \%$ & $14.8 \%$ & \\
\hline & \multirow{2}{*}{$>2$} & $\mathbf{N}$ & 13 & 18 & \\
\hline & & $\%$ & $48.1 \%$ & $66.7 \%$ & \\
\hline \multirow{2}{*}{\multicolumn{2}{|c|}{ Total }} & $\mathbf{N}$ & 27 & 27 & \\
\hline & & $\%$ & $100.0 \%$ & $100.0 \%$ & \\
\hline
\end{tabular}

Table (2): Pathology finding distribution between groups

\begin{tabular}{|c|c|c|c|c|c|c|}
\hline & & & \multicolumn{2}{|c|}{ Group } & \multirow{2}{*}{ Total } & \multirow{2}{*}{$\mathbf{P}$} \\
\hline & & & ERP Group (N=27) & Control group $(\mathrm{N}=27)$ & & \\
\hline \multirow{6}{*}{ Pathology } & \multirow{2}{*}{ Cervical } & $\mathbf{N}$ & $\overline{4}$ & 2 & 6 & \multirow{6}{*}{0.66} \\
\hline & & $\%$ & $14.8 \%$ & $7.4 \%$ & $11.1 \%$ & \\
\hline & \multirow{2}{*}{ Endometrial } & $\mathbf{N}$ & 10 & 10 & 20 & \\
\hline & & $\%$ & $37.0 \%$ & $37.0 \%$ & $37.0 \%$ & \\
\hline & \multirow{2}{*}{ Ovarian } & $\mathbf{N}$ & 13 & 15 & 28 & \\
\hline & & $\%$ & $48.1 \%$ & $55.6 \%$ & $51.9 \%$ & \\
\hline \multirow{2}{*}{\multicolumn{2}{|c|}{ Total }} & $\mathbf{N}$ & 27 & 27 & 54 & \\
\hline & & $\%$ & $100.0 \%$ & $100.0 \%$ & $\begin{array}{c}100.0 \\
\%\end{array}$ & \\
\hline
\end{tabular}

Table (3): Complications and outcome distribution between the two groups

\begin{tabular}{|c|c|c|c|c|c|c|}
\hline & & & \multicolumn{2}{|c|}{$\overline{\text { Group }}$} & \multirow[b]{2}{*}{ Total } & \multirow[b]{2}{*}{$\mathbf{P}$} \\
\hline & & & $\begin{array}{c}\text { ERP Group } \\
(\mathrm{N}=27)\end{array}$ & $\begin{array}{c}\text { Traditional } \\
\text { group }(\mathrm{N}=27)\end{array}$ & & \\
\hline \multirow{4}{*}{ Complications } & \multirow{2}{*}{ None } & $\overline{\mathbf{N}}$ & 25 & 24 & $\overline{49}$ & \\
\hline & & $\%$ & $92.6 \% \%$ & $88.9 \% \%$ & $90.7 \%$ & \\
\hline & \multirow{2}{*}{ Complicated } & $\mathbf{N}$ & 2 & 3 & 5 & 0.63 \\
\hline & & $\%$ & $7.4 \%$ & $11.1 \%$ & $9.3 \%$ & \\
\hline \multirow{4}{*}{ ICU admission } & \multirow{2}{*}{$-V E$} & $\mathbf{N}$ & 27 & 26 & 53 & \\
\hline & & $\%$ & $100.0 \%$ & $96.3 \%$ & $98.2 \%$ & \\
\hline & \multirow{2}{*}{$+\mathrm{VE}$} & $\mathbf{N}$ & 0 & 1 & 1 & 0.31 \\
\hline & & $\%$ & $0.0 \%$ & $3.7 \%$ & $1.8 \%$ & \\
\hline \multirow{4}{*}{ Re admission } & \multirow{2}{*}{$-V E$} & $\mathbf{N}$ & 27 & 27 & 54 & \\
\hline & & $\%$ & $100.0 \%$ & $100.0 \%$ & $100.0 \%$ & 1.00 \\
\hline & \multirow{2}{*}{$+\mathrm{VE}$} & $\mathbf{N}$ & 0 & 0 & 0 & \\
\hline & & $\%$ & $0.0 \%$ & $0.0 \%$ & $0.0 \%$ & \\
\hline \multirow{2}{*}{\multicolumn{2}{|c|}{ Total }} & $\mathbf{N}$ & 27 & 27 & 54 & \\
\hline & & $\%$ & $100.0 \%$ & $100.0 \%$ & $100.0 \%$ & \\
\hline
\end{tabular}


Table (4): Lab parameters distribution between groups

\begin{tabular}{|l|c|c|c|}
\hline & ERP Group $(\mathbf{N}=\mathbf{2 7})$ & Traditional group $(\mathbf{N}=\mathbf{2 7})$ & P \\
\hline \hline HB level $(\mathbf{g m} / \mathbf{d l})$ & $11.82 \pm 0.56$ & $11.74 \pm 0.71$ & 0.660 \\
\hline PLT count ${ }^{* 1000}$ & $236.74 \pm 30.3$ & $248.96 \pm 38.9$ & 0.181 \\
\hline
\end{tabular}

Table (5): Different time's distribution between groups

\begin{tabular}{|l|c|c|c|}
\hline & ERP Group (N=27) & Control group (N=27) & P \\
\hline \hline Catheterization period (hours) & $8.0 \pm 1.46$ & $18.03 \pm 3.1$ & $<0.001^{* *}$ \\
\hline Operative time (minutes) & $189.25 \pm 31.7$ & $198.55 \pm 21.89$ & 0.0987 \\
\hline Movement time (hours) & $11.59 \pm 1.11$ & $24.4 \pm 1.3$ & $<0.001^{* *}$ \\
\hline Hearing sound (hours) & $8.37 \pm 1.27$ & $18.55 \pm 1.25$ & $<0.001^{* *}$ \\
\hline Oral intake (hours) & $9.44 \pm 1.15$ & $20.51 \pm 1.39$ & $<0.001^{* *}$ \\
\hline Time until discharge (hours) & $38.29 \pm 4.95$ & $68.44 \pm 6.5$ & $<0.001^{* *}$ \\
\hline
\end{tabular}

Table (6): Opiate needed distribution between groups

\begin{tabular}{|c|c|c|c|}
\hline & ERP Group $(\mathbf{N}=\mathbf{2 7})$ & Control group $(\mathbf{N}=27)$ & $\mathbf{P}$ \\
\hline \hline Opiates need & $1.37 \pm 0.54$ & $4.9 \pm 1.05$ & $0.00^{* *}$ \\
\hline
\end{tabular}

Table (7): Pain and satisfaction scores distribution between groups

\begin{tabular}{|l|c|c|c|}
\hline & ERP Group $(\mathbf{N}=27)$ & Control group $(\mathbf{N}=27)$ & P \\
\hline \hline Pain score & $2.88 \pm 0.75$ & $5.07 \pm 0.82$ & $<0.0001$ \\
\hline Satisfaction score & $7.18 \pm 0.73$ & $5.81 \pm 0.73$ & $<0.0001$ \\
\hline
\end{tabular}

\section{DISCUSSION}

In this study, age ranged from $23-80$ years with mean age of $51.25 \pm 16.1$ years in group $A$ and a mean age of $56.55 \pm 11.94$ years in group B without any significant difference between the two groups. In addition, the mean body mass index BMI [calculated as weight $(\mathrm{kg}) /$ height $\left.\left(\mathrm{m}^{2}\right)\right]$ was $26.09 \pm 2.19$ for group A and $25.54 \pm 2.36$ for group $\mathrm{B}$ and there was no significant difference between the two groups.

In the pre-operative laboratory investigations, the mean $\mathrm{HB}$ concentration was $11.82 \pm 0.56 \mathrm{gm} \%$ for group A and $11.74 \pm 0.71 \mathrm{gm} \%$ for group B with no significant difference between the two groups. Concerning platelet count, the mean was $236.74 \pm 30.3$ for the group A and $248.96 \pm 38.9$ for group B with no significant difference between the two groups.

In the preoperative period, the patients of the study group was instructed to omit food intake 6 hours prior to the operation and the drinking was continued up to 2 hours before the surgery as the current practice guidelines for obstetric anesthesia from the American Society of Anesthesiologists (ASA). In the control group, food and drinks were prohibited up to 6 hours before the operation was done.

The type of cancers distribution between the two groups showed that ovarian cancer was $48.1 \%$ (13 patients) in group A vs $55.9 \%$ (15 patients) in group B, cervical cancer $14.8 \%$ (4 patients) in group A vs $7.4 \%$ (2 patients) in group B and endometrial cancer was the same in both groups $37.0 \%$ (10 patients).
The pre and postoperative care in both groups, patients were given antibiotics according to guidelines to prevent wound infections as discussed by Morrill $\boldsymbol{e t}$ al. (8) and Nelson et al. (9) and the thromboprophylactics were administrated according to guidelines discussed by Gadducci $\boldsymbol{e t}$ al. ${ }^{\left({ }^{(10)}\right.}$. No data was drawn to compare between both groups for any significant difference.

According to Han-Geurts et al. ${ }^{(11)}$ in gynecology and gynecologic oncology populations, early enteral intake was associated with a faster return of bowel function and a decreased length of stay without an increase in postoperative complications. In the current study, patients were instructed to begin oral intake as soon as possible in the study group and after removal of NG tube and presence of bowel sounds and movement in the control group. The mean time until the first oral intake was $9.44 \pm 1.15$ hours in group A and $20.51 \pm$ 1.39 hours in group B and there was highly significant difference between the two groups without any draw backs on the patients. These results are in agreement with Renaud et al. ${ }^{(12)}$ who reported that patients who resumed a diet before the second postoperative day were significantly higher in the ERAS group ( $94 \%$ vs. $81 \%, \mathrm{P}=0.01)$. Also, Minig et al. ${ }^{(13)}$ showed that eighty-nine percent of the patients in the early oral postoperative feeding (EOF) group were able to resume solid oral intake on the first postoperative day with no statistically significant difference in the incidence of nausea and vomiting compared with traditional oral 
feeding (TOF) group. Fifty-eight percent of the patients in the TOF branch expressed their desire to resume oral feeding earlier, also the mean level of overall postoperative satisfaction was significantly higher in patients of EOF (P <.001). However, Kalogera et al. ${ }^{(6)}$ reported that it is important to note that early feeding is associated with a higher rate of nausea but not vomiting, abdominal distension, or nasogastric tube use. Patient satisfaction with control of vomiting in one series was over $90 \%$ with early feeding despite a higher incidence of nausea in the enhanced recovery group.

In this study, intestinal movement resumption and hearing intestinal sounds were the most important concerns where the mean time until intestinal sounds resumption was $8.37 \pm 1.27$ hour in group $\mathrm{A}$ and 18.55 \pm 1.25 hours in group B with highly significant difference between the two groups. These results are concordant with what Kalogera et al. ${ }^{\left({ }^{(6)}\right.}$ who published that women in the case group had a 1-day earlier for returning of bowel function compared to the historic controls (P,.001). But, these are in contrast to what Macmillan ${ }^{(14)}$ has published earlier in his study where he found that first bowel movement reported [2.8 60.7 versus 2.261 .2 days (P 5 .07)], early versus late feeding groups, respectively, were similar between groups. Also this study is in contrast to what Minig et al. ${ }^{(13)}$ who published that there were no significant differences in term of intestinal bowel recovery between the groups except for the time of passage of flatus, which was faster in patients who received EOF $(\mathrm{P}=0.034)$.

Traditional teaching and intuition suggest that early mobilization decreases pulmonary complications such as atelectasis, decreases insulin resistance, prevents loss of muscle mass, and shortens the interval of return of bowel function. Conversely, immobilization is associated with increased risk of thromboembolism and decreased oxygen delivery to tissues as discussed by Vlug et al. ${ }^{(15)}$. In the current study, early mobilization of patients was instructed where the mean time until first patient ambulation was $11.59 \pm 1.11$ hour in group A and $24.4 \pm 1.3$ hours in group B with a significant difference between the two groups. these results are in agreement was the results of Nikodemski et al. ${ }^{(16)}$ where they found that post-operative early mobilisation on the day of surgery was achieved in $45 \%$ of the study group patients. On the other hand, none of the control group patients mobilised on the day of the operation ( $p$ $<0.0001$ ). In regards to eating post-operatively, $13 \%$ of patients in the study group compared to $1 \%$ in the control group had the first meal on the day of surgery. This rate was increased on the first postoperative day, with $79 \%$ of the study group compared to $8 \%$ in the control group $(\mathrm{p}<0.0001)$.

Postoperative pain analgesia in the study group, multi-modal analgesia consisting of NSAIDs and paracetamol combined with opiates were used in breakthrough pain episodes not responding to analgesia for 2 hours in addition to local infiltration of the incision line with bupivacaine. While in the control group, opiates analgesia with either NSAIDs or paracetamol only were used. In this study, the mean amount of opiates used was $1.37 \pm 0.54$ ampoule morphine in group A and $4.9 \pm 1.05$ ampoule morphine in group B with a significant difference between the two groups. These results are concordant with what Kalogera et al. ${ }^{(6)}$ published where they found that women in the case group received significantly less opioids ( $80 \%$ reduction in first 48 hours after return to room) with an increase in the use of scheduled nonsteroidal anti-inflammatory drugs, acetaminophen, and tramadol. Patient-controlled analgesia was infrequently required in the women in the case group compared to the historic controls.

The pain score of the patients in this study was assessed using the universal pain assessment tool for patients in the two groups where the scale is from (1 to 10 ) in which (1) mean no pain at all and (10) mean severe agonizing un-tolerable pain. The mean pain score was $2.88 \pm 0.75$ for group A and $5.07 \pm 0.82$ for group $\mathrm{B}$ with significant difference between both groups. These results are similar to those published earlier that ERPS patients reported lower maximum pain scores in the post-anesthesia care unit (three vs six $\mathrm{P}<0.0001$ ) and on postoperative day 1 (four vs six; $\mathrm{P}$ $=0.002$ ). These results are in contrast to what Minig $\boldsymbol{e t}$ al. ${ }^{(13)}$ has published that postoperative median pain score was similar in both groups. Also, these results are in contrast to what has published that immediate postoperative pain scores did not differ significantly between patients in the case group and patients in the control group on postoperative day 0 (2.4 compared with $2.6, \mathrm{P}=.24$ ).

In the current study, Satisfaction scores of the patients was done using a scale from 0-10 where (0) was not satisfied at all and (10) was very satisfied from which the overall mean satisfaction score was concluded to be $7.18 \pm 0.73$ in group A and $5.81 \pm 0.73$ in group B withj a significant difference between the two groups. These results are concordant with what was published by Ottesen et al. ${ }^{(17)}$ where in their study, $92.7 \%$ of the patients stated that their hospitalization was "as expected," "easier than expected," or "much easier than expected." Most patients were satisfied with their hospital length of stay (LOS). Only a small percentage of patients of less than 5\% (2/41) reported feeling "a little pressure put on them toward discharge," among which one was discharged on postoperative day 8. In a 0 to 10 scale of "how acceptable the program and advice had been," the median score was 10 . Patient satisfaction rates have universally been reported high ranging from $75 \%$ to $95 \%$ across studies. Also in the

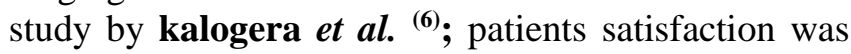
high in all studied aspects of perioperative care 
including patient education, quality of care during hospitalization, the discharge process, and pain management with $90-90 \%$ rating satisfaction as excellent or very good. In contrast to what Polle $\boldsymbol{e t}$ al. (18) has concluded in their study where they stated that "Total patients' satisfaction score was comparable in both groups ( 50.4 and 49.8 of a potential 80 points in the FT and TC groups, respectively; $\mathrm{p}=0.84$ )".

Hospital stay reduction is also one of the goals of ERPS protocols where in this study the mean of length of stay was found to be $38.29 \pm 4.95$ hours in group A and $68.44 \pm 6.5$ hours in group B indicating significant difference between the two groups of the patients. These results are in agreement with the results that published by Nelson et al. ${ }^{(9)}$ which showed a decrease of the median LOS (2.5 days ( 0 to 11 ) vs. 3 days ( 1 to $14) ; p=0.002)$ and proportion of discharged patient at target LOS of 2 days ( $45 \%$ vs. $24 \%$; $\mathrm{p}=0.002$ ).

\section{CONCLUSIONS}

Implementation of enhanced recovery pathways (ERAS) in gynecological oncologic surgery was associated with an overall improvement in postoperative outcomes. The implementation of a successful ERAS program led to early ambulation, early oral intake, earlier return of intestinal sounds, decreasing time of catheterization, decreasing incidence of PONV and IONV, acceptable pain management with reduced opioids, reducing length of stay with stable readmission and morbidity rates, good patient satisfaction, and substantial cost reduction.

\section{REFERENCES}

1. Kehlet H (1997): Multimodal approach to control postoperative pathophysiology and rehabilitation. $\mathrm{Br} \mathrm{J}$ Anaesth., 78: 606-17.

2. Dowdy S, Borah B, Bakkum-Gamez J et al. (2012): Prospective assessment of survival, morbidity, and cost associated with lymphadenectomy in lowrisk endometrial cancer. Gynecol Oncol., 127: 5-10.

3. Bristow R, Tomacruz R, Armstrong D et al. (2002): Survival effect of maximal cytoreductive surgery for advanced ovarian carcinoma during the platinum era: a meta-analysis. J Clin Oncol., 20: 1248-59.

4. Van der Zee A, Oonk M, De Hullu J et al. (2008): Sentinel node dissection is safe in the treatment of earlystage vulvar cancer. J Clin Oncol., 26: 884-9.
5. Steenhagen $\mathbf{E}$ et al. (2016): Enhanced recovery after surgery: it's time to change practice. Nutr Clin Pract., 31: 18-29.

6. Kalogera E, Bakkum-Gamez J, Jankowski C et al. (2013): Enhanced recovery in gynecologic surgery. Obstet Gynecol., 122: 319-28.

7. Kehlet H, Wilmore D (2002): Multimodal strategies to improve surgical outcome. Am J Surg., 183: 630-41.

8. Morrill M, Schimpf M, Abed H et al. (2013): Antibiotic prophylaxis for selected gynecologic surgeries. International Journal of Gynecology \& Obstetrics, 120 (1): 10-15.

9. Nelson G, Altman A, Nick A et al. (2016): Guidelines for pre- and intra-operative care in gynecologic/oncology surgery: Enhanced Recovery after Surgery (ERAS) Society recommendations-Part I. Gynecol Oncol., 140: 313-22.

10.Gadducci A, Cosio S, Spirito $\mathrm{N}$ et al. (2010): The perioperative management of patients with gynaecological cancer undergoing major surgery: a debated clinical challenge. Critical Reviews in Oncology/Hematology, 73 (2): 126-140.

11. Han-Geurts I, Hop W, Kok N et al. (2007): Randomized clinical trial of the impact of early enteral feeding on postoperative ileus and recovery. Br J Surg., 94 (5): 55561.

12. Renaud M, Bélanger L, Lachapelle $P$ et al. (2019): Effectiveness of an Enhanced Recovery After Surgery Program in Gynaecology Oncologic Surgery: A SingleCentre Prospective Cohort Study. Journal of Obstetrics and Gynaecology Canada, 41 (4): 436-42.

13. Minig L, Biffi R, Zanagnolo $V$ et al. (2009): Reduction of postoperative complication rate with the use of early oral feeding in gynecologic oncologic patients undergoing a major surgery: a randomized controlled trial. Annals of Surgical Oncology, 16 (11): 3101-6.

14. MacMillan S (2000): Early feeding and the incidence of gastrointestinal symptoms after major gynecologic surgery. Obstetrics \& Gynecology, 96 (4): 604-8.

15. Vlug M, Bartels S, Wind J et al. (2012): Which fast track elements predict early recovery after colon cancer surgery?. Colorectal Disease, 14 (8): 1001-8.

16. Nikodemski T, Biskup A, Taszarek A et al. (2017): Implementation of an enhanced recovery after surgery (ERAS) protocol in a gynaecology department-the followup at 1 year. Contemporary Oncology, 21 (3): 240-6.

17. Ottesen M, Sorensen M, Rasmussen Y et al. (2002): Fast track vaginal surgery. Acta Obstet Gynecol Scand., 81:138-46.

18. Polle S, Wind J, Fuhring J et al. (2007): Implementation of a Fast-Track Perioperative Care Program: What Are the Difficulties? Digestive Surgery, 24 (6): 441-9. 